\title{
Pilot autonomous hybrid hydrogen refueling station utilizing a metal hydride compressor covering local transportation needs
}

\author{
Nikolaos Chalkiadakis ${ }^{1, *}$, Athanasios Stubos ${ }^{1}$, Emmanuel I. Zoulias, and Emmanuel Stamatakis ${ }^{2}$ \\ ${ }^{1}$ NCSR "DEMOKRITOS", Athens, Greece \\ ${ }^{2}$ New Energy \& Environmental Solutions \& Technologies - NEEST, Athens, Greece \\ ${ }^{3}$ Institute of Petroleum Research / Foundation for Research and Technology - Hellas (IPR/FORTH), Chania, Greece
}

\begin{abstract}
The need for decreasing carbon emissions in the transportation sector in order to meet the targets of the European Union by 2030, inevitably leads to the large scale adoption of cleaner alternatives. Hydrogen fueled vehicles could possibly provide one such alternative, if we could assume that the necessary infrastructure would be widely available throughout Europe. Already, the European Union has committed to the construction of a significant number of Hydrogen Refueling Stations (HRS) by year 2025 and in view of that, there is a need of developing suitable configurations for the production, compression, storage and dispensing of green hydrogen to hydrogen fueled vehicles. This work presents an autonomous hybrid system which produces green hydrogen by PV- powered water electrolysis (PEM), which is subsequently compressed by a novel metal hydride hydrogen compressor to pressures up to 200 bar. This pilot HRS will meet the daily demand of 2 scooters and a golf cart which have been transformed, in order for their electric motor to be powered by a hydrogen fuel cell instead of a battery. An important element of the work which is presented, revolves around the integration of the metal hydride compressor with the rest of the system, and how this integration won't hinder its functionality. The complete system design and layout is presented, while the results from the system operation could give a good idea regarding the optimal system sizing for similar large scale applications.
\end{abstract}

\section{Introduction}

Worldwide, there is an obvious effort for the minimization of the gas emissions which are produced by all aspects of human activity. The main reason for this is the increasing presence of the effects of climate change which are already starting to be felt, which are in large due to carbon dioxide emissions (Fig.1).

The above mentioned effort is evident if one looks at the relevant decisions of most governments, the most notable example being the targets defined by the European Union for the decrease in greenhouse gas (GHG) emissions by 2030 and 2050.

More specifically, the E.U. has defined its 2030 targets as:

- $40 \%$ reduction in GHG emissions in relation to the 1990 levels

- At least $32 \%$ renewable energy share

- At least 32.5\% improvement in energy efficiency

Currently, one of the sectors of human activity with the most GHG emissions is the transportation sector. Although common sense would lead to the expectation of decreasing levels of emissions due to measures similar to those which are mentioned above, the reality is that they remain relatively the same. This fact can be attributed to several causes, the most likely of which are:
- The trend of consumers selecting larger and heavier vehicles (not just in the USA but in Europe and Asia as well). This trend is in part counterbalanced by the increasing penetration of electric vehicles and the subsequent decrease of the share of Diesel vehicles.

- The growth of the global GDP, combined with the rise of e-commerce which requires fast product delivery, resulting in the increase of emissions due to transportation of goods.

The above lead to the realization of the importance of the transportation sector in shaping the worldwide GHG emissions and for this reason the E.U. has set specific targets to be met by years 2030 and 2050 . These are

- $30 \%$ decrease of $\mathrm{CO}_{2}$ emission by 2030 and

- $60 \%$ decrease of $\mathrm{CO}_{2}$ emissions by 2050

In order meet these targets, hydrogen could be the fuel of choice for the transportation sector, but in order for it to have an expanded role as a fuel in the future, an extensive refueling network must be developed. There is also the counter-argument that without a sufficient number of hydrogen fueled vehicles, refueling infrastructure is not financially viable, which leads to a chicken and egg problem [1]. A way to avoid this problem is the gradual development of stations which could meet the demand for hydrogen of smaller vehicle fleets, or of vehicles such as scooters, hydrogen bicycles 


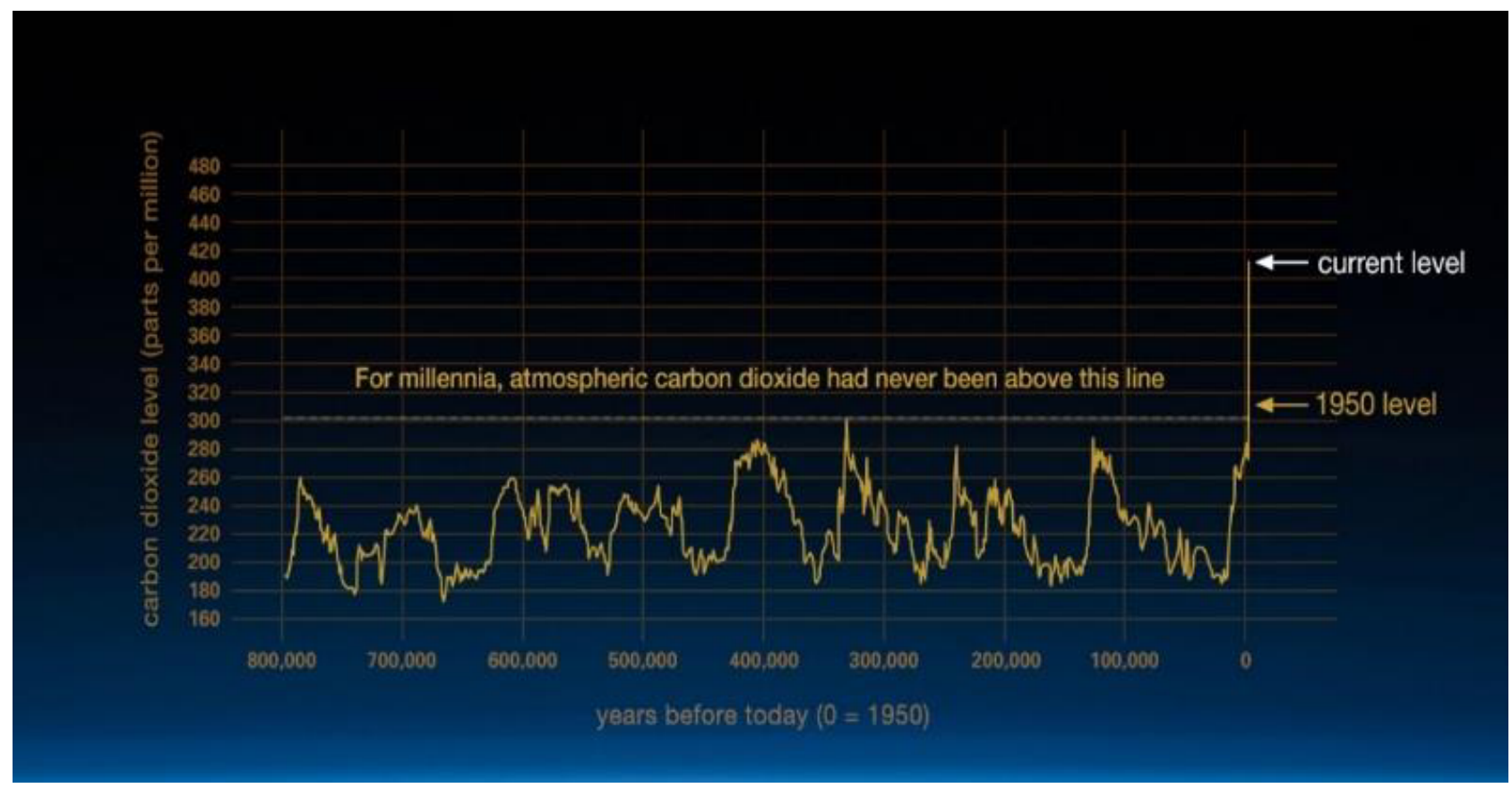

Fig. 1. Carbon Dioxide levels over the years (climate.nasa.gov)

and other light duty vehicles, eventually expanding to larger automotive applications once the financial viability of the smaller stations has been showcased.

An added benefit of this scenario is the acquisition of the relevant knowhow through numerous projects and different configurations, leading to the identification of the optimal case specific layout for future installations.

The project which will be analyzed throughout this paper, aims to showcase the design and construction of the first autonomous HRS for hydrogen fueled vehicles in Greece.

\section{The HRS subsystems}

The pilot refueling station which has been developed for the purpose of the H2TRANS project is designed to cover the daily hydrogen demand of two scooters and a golf cart, both of which have been configured in a way that their electric motors are powered by hydrogen fuel cells instead of batteries. As a result the whole system has been sized accordingly. The components that the system is comprised of are the following:

- Water treatment unit

- PV panels

- PEM water electrolysis

- Buffer and Low/High pressure hydrogen tank

- Metal Hydride Compressor

- Hot/Cold water tanks for the compressor function

- Hydrogen Dispenser

The above components are interconnected by a gas network which consists of various valves, flow regulators, flow meters, pressure/temperature transmitters and vents. In the following paragraphs, the exact role and specifications of each component will be detailed.

\subsection{Water treatment}

Water quality is an important aspect of a PEM electrolyzer's function and the threshold in which it can operate is strictly determined by the manufacturer. The most important characteristic for the water used in PEM electrolysis is its high resistivity, which is achieved by low content in contaminants such as electrolytes, ions, sodium and chlorides. Such contaminants are removed in the water treatment unit by filtering and deionizing water by using mixed bed resins and carbon filters. Also in order to desterilize water a UV lamp is used, which kills bacteria and microorganisms. The water which has passed though the treatment unit enters the electrolysis with an electrical conductivity lower than $0.1 \mu \mathrm{S}$ and concentration of sodium and chlorides lower than

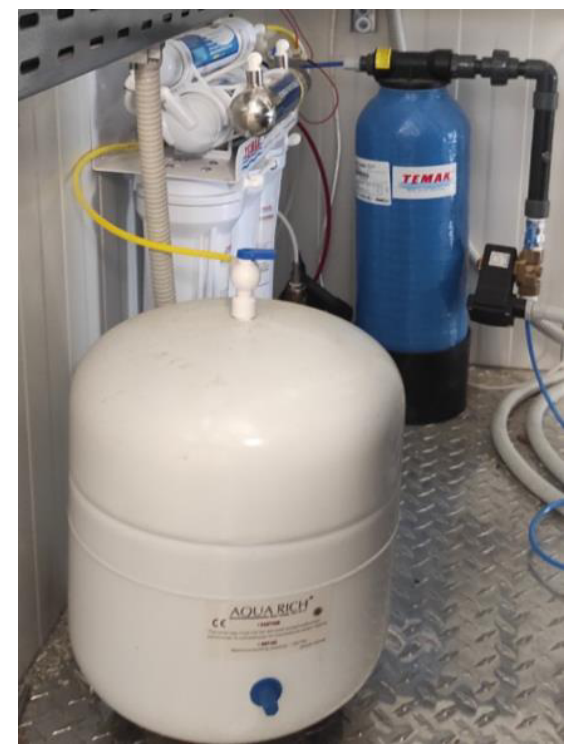

Fig. 2. Water Treatment 
$5 \mu \mathrm{g} / \mathrm{L}$. The unit which is used in this case is depicted in Figure 2.

\subsection{Hydrogen Production}

Despite hydrogen being the most abundant element in the universe, the only form in which it can be found on earth is as part of a compound with other elements. Thus in order to isolate it for the purposes of using it as fuel, certain chemical reactions should be utilized, which depend on the compound that hydrogen is found in. The compounds in which hydrogen is most frequently found in are hydrocarbons (be it fossil fuels or biomass) and water. The most common methods for producing hydrogen through hydrocarbons are steam methane reforming (SMR), thermo cracking (TC), partial oxidation (POX) and coal gasification (CG). For the case of water electrolysis, the main methods for the production of hydrogen are PEM and alkaline electrolysis, while different approaches include photoelectrolysis (PHE) or photolysis, water thermolysis (WT), and photobiological processes [2].

The hydrogen production unit of the installation has been selected to be a PEM electrolysis unit. The reasoning behind choosing PEM electrolysis as a means of hydrogen production is the fact that it presents an ideal solution for coupling with renewable sources in order to produce green hydrogen. After leaving the water treatment unit, water enters the PEM electrolysis (Figure 3) which is made of three $1.3 \mathrm{~kW}$ stacks, each of which produces hydrogen at 16 bar at a purity greater than $99.99999 \%$. The maximum flow rate of each stack is $3300 \mathrm{cc} / \mathrm{min}$ or $0.714 \mathrm{~kg} / \mathrm{h}$ in terms of mass flow with a mean output of $0.127 \mathrm{~kg} / \mathrm{h}$ throughout the day. The electrolysis unit has a modular design that allows for the installation of more stacks in order to increase the hydrogen flow rate. In its current size though, the daily amount of hydrogen produced can meet the respective demand of the vehicles used in the project.

\subsection{Hydrogen storage}

There are several ways to store hydrogen, some of which are pretty mainstream and have been used numerous times and some which are being currently tested and evaluated. A critical parameter for the assessment of a storage technology has to do with the suitability of the materials which are used, its volumetric density, as well as the reversibility of uptake and release [3]. The most popular storage methods, are compressed gas, liquefied and metal hydrides.

For the purposes of this project, hydrogen is stored as a compressed gas. The storage system (Figure 4) is divided in two parts, one used as a buffer and dedicated for low pressure storage at 16 bar. Basically, hydrogen flowing out of the electrolysis unit is stored in the low pressure compartment before entering the compressor. The low pressure storage ensures that regardless of whether the electrolysis operates or not, there is a sufficient quantity of hydrogen to feed the compressor.
After compression hydrogen is stored at the second compartment at the pressure of 200 bar. Both compartments have a total volume of $300 \mathrm{~L}$, while the tanks are made of steel.

\subsection{Hydrogen Compression}

Hydrogen compression constitutes the main barrier regarding the large scale adoption of hydrogen as a fuel, not just for transportation applications but for stationary applications as well. For this reason, improvements in the efficiency, durability and reliability of hydrogen compressors is of utmost importance. Currently, the most commonly used hydrogen compression technologies in HRSs are based on mechanical compression. These include either reciprocating or diaphragm compressors, both of which despite being relatively mature technologies, have several drawbacks, most of which stem from the presence of moving parts. The most notable related issues are [4]

a) the high mechanical stresses which unavoidably lead to the need for lower speeds, which in turn leads to relatively low flow rates

b) high manufacturing and maintenance costs

c) high noise and vibration

d) potential diaphragm rupture [5]

We are thus lead to the conclusion that there exists the need for identifying and developing different solutions for hydrogen compression, which will tackle all the above issues in an efficient way. The most promising alternatives for hydrogen compressors include ionic liquid, metal hydride and electrochemical compressors, all of which will be discussed in the following paragraphs.

The compressor technology used in this case is the Metal Hydride compressors (MHC). MHCs base their operation on the property of metal hydrides of absorbing/desorbing hydrogen depending on their temperature, thus they are classified as thermal compressors. Compression is achieved by a reversible heat-driven interaction of a hydride forming metal or alloy or intermetallic compound with hydrogen to form $\mathrm{MH}$. Since their operation is based on thermal

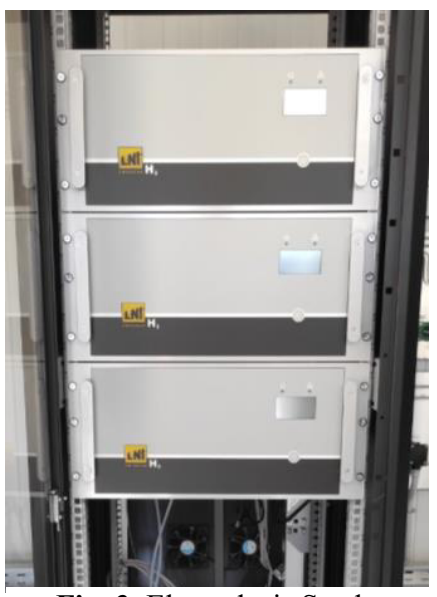

Fig. 3. Electrolysis Stacks

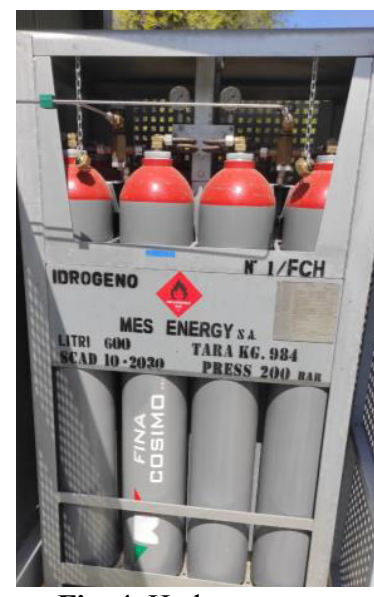

Fig. 4. Hydrogen storage tanks 
processes, they could potentially utilize waste heat and/or excess renewable energy (e.g. solar thermal) for the required heating and cooling of the $\mathrm{MH}$ tanks, which reduces significantly their operational cost and increases the overall efficiency of the system [6].

Metal hydrides which are used in such compressors are special alloys like AB5-type (example, $(\mathrm{La}-\mathrm{Ce})(\mathrm{Ni}-$ $\mathrm{Al})_{5}$ ) or AB2-type (for example, (Ti-Zr)(Mn-Cr-Fe-Co$\mathrm{V})_{2}$ ) which can chemically store hydrogen in their metallic lattice [7]. Notable advantages of $\mathrm{MH}$ compression include their simple design and operation, the absence of moving parts which results in little to noise during operation, as well as their compactness and reliability. Since this specific compression method is used for the purposes of the project, details on its integration will be discussed in the following paragraphs. The compressor used in this installation (Figure 5) is comprised of 6 stages and can reach pressures of up to $220 \mathrm{bar}$, while having as input hydrogen from the electrolysis at 16 bar which can be reduced to the desired input pressure by a pressure regulator. The heating and cooling medium of the compressor stages is water which flows concentrically to the metal hydride bed, through an outer jacket and an inner helical coil. The operation of the compressor can be divided in two phases which are differentiated by the stages of the compressor which are being heated and cooled. So for example, during phase A stages 1, 3 and 5 are cooled, while 2, 4 and 6 are heated.

During phase A, since stage 1 is being cooled, it is therefore adsorbing hydrogen from the buffer tank for the pre-specified time period and at the pre-specified pressure which is needed for the complete saturation of the $\mathrm{MH}$. Once phase A is finished, phase B begins, meaning that the first stage is heated at a pre-specified temperature for as long as it takes for the complete desorption of the stored hydrogen while at the same time stage 2 is being cooled, absorbing in the process hydrogen leaving stage 1 . What needs to be taken into account in the design of the compressor is that each stage's inlet pressure is the previous stage's outlet pressure, hinting at the desired characteristics of the MH which will be used. The same process is repeated until hydrogen leaves the sixth stage at the pressure of 200 bar and the steady state of the compressor begins.

As mentioned, the $\mathrm{MH}$ compressor achieves compression by providing hot and cold water to the appropriate stages during each phase, there is therefore a constant need for water of the desired temperatures. The matter of temperature is of great importance, since $\mathrm{MH}$ have a specific range of temperatures in which they operate optimally. In this case, the system utilizes a heat pump which simulates the waste heat flow from an industry or the hot water flow from a solar thermal/chiller unit. The sizing of the heating/cooling system is determined by the thermal needs of the MHC stages, which are calculated by taking into account the desired temperatures of the water inside the stages as well as the exothermic/endothermic nature of adsorption/desorption processes respectively. In order to estimate the mass flow of the heating/cooling medium, what should be taken into account is the mass of the $\mathrm{MH}$ and its reaction enthalpy, while also important are the heat capacity of the $\mathrm{MH}$ and of the hydride bed walls. Once all the above have been calculated for all stages of the MHC, one can estimate the required thermal energy which needs to be provided/ extracted to each stage. This can then be translated to mass flow for the heating/cooling medium leading to the optimal sizing of the hot and cold water tanks as well as of the required pumps for the medium circulation (Figure 6).

For the automation of the compression and of the station's function in general, the installation is monitored and controlled by a PLC unit. By monitoring the system's status constantly (temperatures and pressures of the complete gas network), the controller ensures the smooth operation of the installation at all

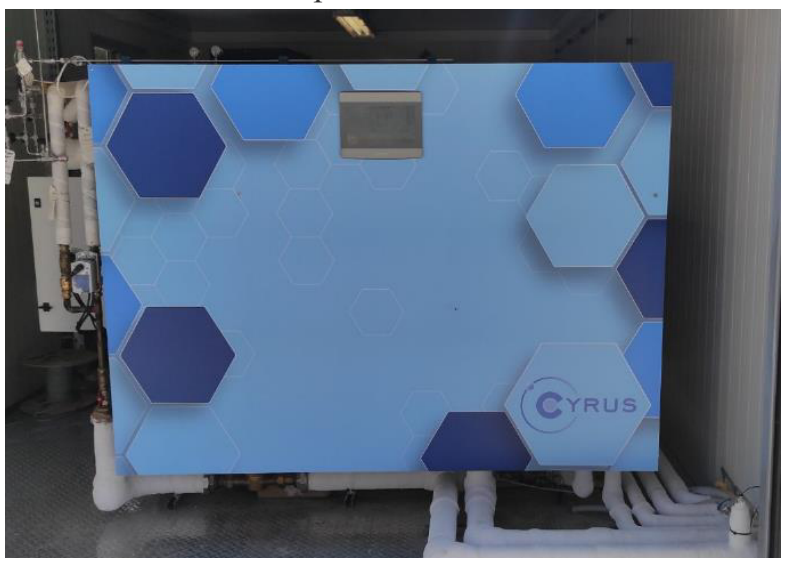

Fig. 5. Metal Hydride Compressor

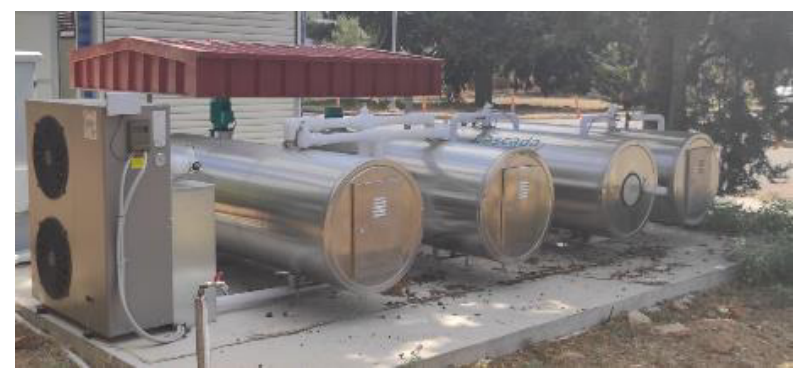

Fig. 6. Hot and cold water tanks and pumps for the compressor operation

times. The automated function of the compressor is ensured by the control unit and an algorithm of successive phase transitions. The systems begins to operate only when the temperature transmitters inform the controller that the water temperature is in its prespecified temperature. The controller then, basically switches on and off the appropriate valves which regulate the flow of water and when they are at the correct state (open or closed), it gives a signal to the pumps to start operating. The system then runs for the 
pre-specified time and stops only to switch phases or in case of a detected abnormality, such as an overpressure, or the absence of the desired temperature.

\subsection{Hydrogen Dispenser}

After being compressed to 200 bar, the high pressure hydrogen is stored in the pressurized gas tanks which have been shown, where it is stored until a vehicle needs to be refilled. In this case, the dispenser is fitted to the vehicle's tank and the PLC initiates the refueling algorithm. Basically what happens is the comparison of the pressure inside the vehicles tank and the station's supply pressure. As long as the former is lower than the latter, hydrogen will flow to the vehicle's tank. Once the two are equal, the dispensing halts, and the dispenser is detached from the vehicle. In the process, the system has gathered data about the quantity of hydrogen which was dispensed, the time required as well as its hypothetical cost.

\subsection{Gas Network}

An integral part of the installation, one which ensures the automation of the system as well as its adequate monitoring at all times is the gas network (Figure 7). In Fig. 7 one can see the way hydrogen flows through the various components of the network. These include solenoid, hand and check valves, pressure and temperature transmitters and indicators, pressure regulators and a flow meter. Also there are numerous configurations for venting hydrogen to the atmosphere in order to increase the system's safety. The gas network can be divided to three parts, namely the low pressure and high pressure compartments and the dispensing compartment, which can be seen in fig $\mathrm{x}$, at the bottom, upper and middle parts of the panel respectively. The low pressure compartment is responsible for monitoring the pressure of hydrogen which builds up in the low pressure tank, with the ultimate purpose of facilitating its input to the compressor, at the pressure set by the regulator. The same goes for the high pressure compartment, with the difference that it feeds the high pressure tanks. The dispensing compartment regulates the pressure of hydrogen leaving the high pressure storage and finally allows the filling of the vehicle that needs refueling, through an algorithm which opens and closes the right solenoid valves, as set by the controller. The whole process is documented by the flow meter, which transmits to the control panel the quantity of the hydrogen which was used.

\subsection{System Integration}

In order to determine whether the system can operate harmoniously, we need to identify potential bottlenecks or shortages. Mean hydrogen production rates have been already addressed and established as 127 grams per hour, while as seen in Diagram 1 the compressor has an output of roughly 100 grams per hour. Note that Diagram 1 shows the compressor output at 3 cycles in which the $6^{\text {th }}$ and final stage is heated and 2 in which it is cooled, with the heating obviously occurring when the mass output increases. The mismatch between the two outputs seems to create a slight bottleneck, which is currently being addressed by experimenting with the compressor's temperature ranges in order to further increase its output. Regardless of that, this bottleneck doesn't seem to hinder the functionality of the system in practical use. The vehicles which are refueled in the station, have a total capacity of 161 grams in terms of wight, meaning that they can be refueled numerous times throughout the day, taking into consideration that they will not require refueling on an hourly basis. Thus it can be assumed that the station could potentially cover the needs of even more vehicles. Regardless of the vehicles which can be served in the installation in its present status, there is the ability of increasing its capacity with only minor changes. This is on the one hand due to the modularity of the electrolysis unit which can increase its output simply by adding more stacks and on the other hand due to the modular design of the compressor which can also increase its output by adding more stages in parallel with the existing ones.

One of the reasons that the installation can have such a smooth function lies in the nature of hydrogen as an energy storage medium. It is obvious that the fluctuations in the electrolysis operation which are due to the fluctuations of the solar irradiance can be

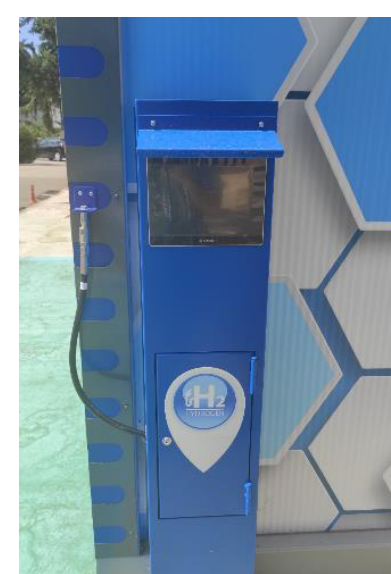

Fig. 7. Hydrogen Dispenser

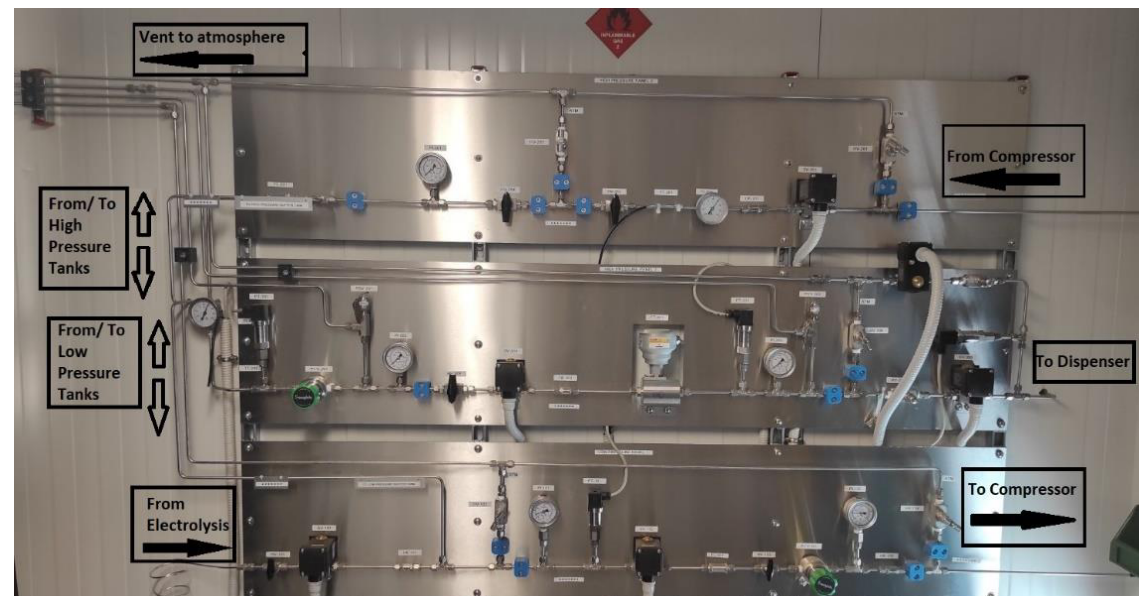

Fig. 7. Gaseous Network Panel. Top to bottom: High pressure panel, Dispensing panel, Low Pressure panel 
counterbalanced by the hydrogen which is already produced and stored during peak hours of solar irradiance. The fact that there is constantly a quantity of hydrogen stored both in the low pressure and high pressure tanks ensures the operation of the system even in times when the electrolysis does not operate, thus gives the ability of refueling vehicles throughout the day.

\section{Conclusions}

The work presented in the previous paragraphs focuses on showcasing the basic aspects of operation of a hydrogen refueling station which makes use of a metal hydride compressor. An important aspect for the system's smooth operation is the matching of the electrolysis and the compressor's flow rates, since it is vital for identifying potential hydrogen bottlenecks or shortages. The installation's hydrogen producing unit is a 4kW PEM electrolysis which was shown to be able to meet the load of 2 scooters and a golf cart on a daily basis. The system proved to be completely automated in terms of hydrogen production and compression, while the only part which needs a human presence is the refueling of the vehicles. Finally the modularity of the system which is based on the modular design of the electrolysis and the compressor leads to the conclusion that a larger number of vehicles could potentially be served in an installation similar to this, with only minor changes on the core infrastructure of the installation.

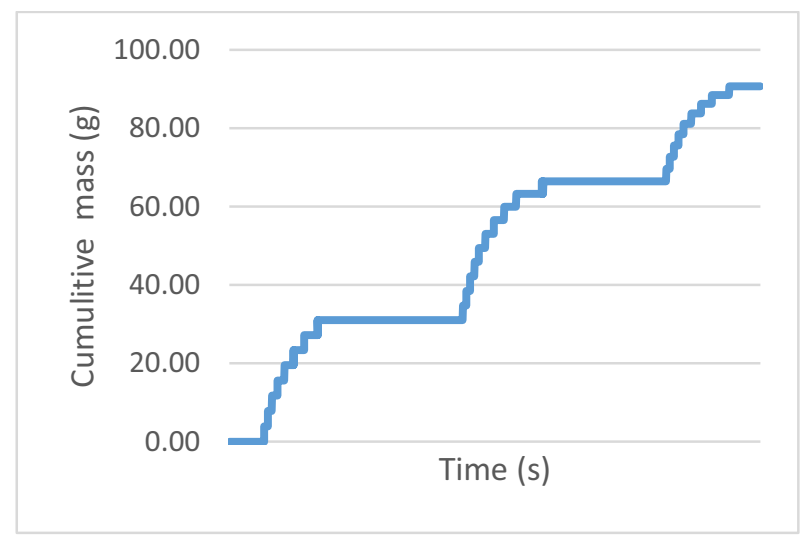

Diagram 1. Compressor mass output

\section{Acknowledgments}

This work is co-financed by the European Regional Development Fund of the EU and Greek national funds under the call RESEARCH - CREATE - INNOVATE (project H2TRANS_T1EDK-05294).

\section{References}

[1] D.L. Greene, J. M. Ogden Z. Lin: "Challenges in the designing, planning and deployment of hydrogen refueling infrastructure for fuel cell electric vehicles", eTransportation, 6, 100086, (2020)
[2] P. Nikolaidis, A. Poullikkas: "A Comparative Overview of Hydrogen Production Processes", Renewable Energy and Sustainable energy reviews, 67, 597-611 (2017)

[3] Andreas Züttel: "Materials for hydrogen storage", Materials Today,6 (9), 24-33(2003)

[4] Almasi, A.: Reciprocating compressor optimum design and manufacturing with respect to performance, reliability and cost. World Acad. Sci. Eng. Technol. 52, 48-53 (2009)

[5] Jiahao, C., Xiaohan, J., Chuang, et al.: Design and validation of new cavity profiles for diaphragm stress reduction in a diaphragm compressor. IOP Conf. Ser. Mater. Sci. Eng. (2015)

[6] F. Laurencelle, Z. Dehouche, J. Goyette, T.K. Bose, "Integrated electrolyser - metal hydride compression system”, Int J. Hydrogen Energy 31, 762-768, 2006.

[7] Bossel U., "Does a Hydrogen Economy Make Sense?", Proceeding of the IEEE, 2006. 